\title{
DIGITALCOMMONS
}

@WAYNESTATE -

Wayne State University

Physics and Astronomy Faculty Research

Publications

Physics and Astronomy

$12-1-1986$

\section{Exact evaluation and recursion relations of two- center harmonic oscillator matrix elements}

P. J. Drallos

Wayne State University

J. M. Wadehra

Wayne State University, ad5541@wayne.edu

\section{Recommended Citation}

Drallos PJ, Wadehra JM. Exact evaluation and recursion relations of two-center harmonic oscillator matrix elements. J. Chem. Phys. 1986;85(11):6524-6529. doi: 10.1063/1.451433

Available at: http://digitalcommons.wayne.edu/phy_astro_frp/104

This Article is brought to you for free and open access by the Physics and Astronomy at DigitalCommons@WayneState. It has been accepted for inclusion in Physics and Astronomy Faculty Research Publications by an authorized administrator of DigitalCommons@WayneState. 


\title{
Exact evaluation and recursion relations of two-center harmonic oscillator matrix elements
}

\author{
P. J. Drallos and J. M. Wadehra \\ Department of Physics and Astronomy, Wayne State University, Detroit, Michigan 48202
}

(Received 16 July 1986; accepted 21 August 1986)

\begin{abstract}
Using vibrational wave functions of two relatively displaced harmonic oscillators of arbitrary frequencies, Franck-Condon overlap integrals and matrix elements of $x^{l}, \exp (-2 c x)$, and $\exp \left(-c x^{2}\right)(x$ is the internuclear separation) are obtained. Useful three-term, four-term, and five-term recursion relations among these matrix elements are derived. It is shown that all of the relevant matrix elements can be obtained from a mere knowledge of the lowest two Franck-Condon overlap integrals. Results are illustrated by computation of Franck-Condon factors for the $A^{1} \Sigma_{u}^{+}-X^{1} \Sigma_{g}^{+}$and the $B{ }^{1} \Pi_{u}-X^{1} \Sigma_{g}^{+}$systems of ${ }^{7} L_{2}$.
\end{abstract}

\section{INTRODUCTION}

A quantitative description of transition probabilities for various vibrational levels (that is, vibrational excitation) as well as of intensities of various lines in the spectra of diatomic and polyatomic molecules requires matrix elements of various powers of the internuclear separation $x$ between vibrational levels belonging to two different electronic states of the molecule. ${ }^{1}$ A Franck-Condon overlap integral is a special example of such a matrix element. For low-lying vibrational levels, the potential curves of the molecular electronic states can be represented with reasonable accuracy by those of linear harmonic oscillators. For higher vibrational levels, where anharmonicity becomes important, the potential of a Morse oscillator is a better representation of the true potential curve. Even in such a case as the Morse oscillator, if one were to use first-order perturbation theory with the linear harmonic oscillator as the zero-order approximation, the matrix elements of powers of $x$ would appear in the correction factors. With this spirit in mind, an attempt is made in this paper to obtain general analytic expressions and simple recursion relations for two-center harmonic oscillator matrix elements of various functions (powers, exponential, and Gaussian) of $x$. In fact, a general five-term recursion relation to be derived below [Eq. (32) ], is valid for any analytical function of $x$ that could be expanded as a power series in $x$.

The evaluation of Franck-Condon factors, which essentially involves an overlap integral between wave functions of vibrational levels belonging to two different electronic states of a molecule, using linear harmonic oscillator wave functions has been carried out in a number of investigations, and proper kudos have been distributed by Waldenstrøm and Razi Naqvi. ${ }^{2}$ Various theoretical methods for obtaining the Franck-Condon factors have been reviewed more recently by Kuz'menko et $a l^{3}{ }^{3}$ Overlap matrix elements of various functions of $x$ using, once again, vibrational wave functions of two different harmonic oscillators, have been analytically obtained in some recent papers. ${ }^{4,5}$ In Sec. IV of the present paper we will obtain some general recursion relations among these matrix elements. A single and double ket notation (for example, $|m\rangle$ and $|n\rangle\rangle$ ) will be used to distinguish between the vibrational eigenfunctions belonging to two different electronic states.

\section{THE FRANCK-CONDON OVERLAP INTEGRAL}

The relevant potential energy curves are replaced by those of one-dimensional harmonic oscillators of frequency $\omega_{1}$ and $\omega_{2}$, with a relative separation of $r$. For convenience, define $\omega_{0}=\hbar\left(\mu r^{2}\right)$, where $\mu$ is the reduced mass of the nuclei. The two-center Franck-Condon overlap integral is then defined as $\langle m \mid n\rangle\rangle$, where

$$
\begin{aligned}
\langle x \mid m\rangle= & \left(2^{m} m !\right)^{-1 / 2}\left[\omega_{1} /\left(\pi \omega_{0} r^{2}\right)\right]^{1 / 4} \\
& \times \exp \left[-\omega_{1} x^{2} /\left(2 \omega_{0} r^{2}\right)\right] H_{m}\left[\left(\omega_{1} / \omega_{0}\right)^{1 / 2} x / r\right]
\end{aligned}
$$

is the wave function of the $m$ th level of the harmonic oscillator associated with the potential $V_{1}=\frac{1}{2} \mu \omega_{1}^{2} x^{2}$, and

$$
\begin{aligned}
\langle\langle x \mid n\rangle\rangle= & \left(2^{n} n !\right)^{-1 / 2}\left[\omega_{2} /\left(\pi \omega_{0} r^{2}\right)\right]^{1 / 4} \\
& \times \exp \left[-\omega_{2}(x-r)^{2} /\left(2 \omega_{0} r^{2}\right)\right] \\
& \times H_{n}\left[\left(\omega_{2} / \omega_{0}\right)^{1 / 2}(x-r) / r\right]
\end{aligned}
$$

is the wave function of the $n$th level of the harmonic oscillator associated with the potential $V_{2}=\frac{1}{2} \mu \omega_{2}^{2}(x-r)^{2}$. Thus,

$$
\begin{aligned}
\langle m \mid n\rangle\rangle= & \frac{\left(\omega_{1} \omega_{2}\right)^{1 / 4}}{\left[\pi \omega_{0} r^{2}\left(2^{m+n} m ! n !\right)\right]^{1 / 2}} \exp \left[\frac{-\omega_{1} \omega_{2}}{2 \omega_{0}\left(\omega_{1}+\omega_{2}\right)}\right] \\
& \times \int_{-\infty}^{\infty} \exp \left\{-\left[\left(\frac{\omega_{1}+\omega_{2}}{2 \omega_{0} r^{2}}\right)^{1 / 2} x\right.\right. \\
& \left.\left.-\frac{\omega_{2}}{\left[2 \omega_{0}\left(\omega_{1}+\omega_{2}\right)\right]^{1 / 2}}\right]^{2}\right\} H_{m}\left[\left(\omega_{1} / \omega_{0}\right)^{1 / 2} x / r\right] \\
& \times H_{n}\left[\left(\omega_{2} / \omega_{0}\right)^{1 / 2}(x-r) / r\right] d x .
\end{aligned}
$$

Now, on changing the integration variable from $x$ to $t=x\left[\left(\omega_{1}+\omega_{2}\right) /\left(2 \omega_{0} r^{2}\right)\right]^{1 / 2}$,

$$
\begin{aligned}
\langle m \mid n\rangle\rangle= & N_{m n} \pi^{-1 / 2} \int_{-\infty}^{\infty} \exp \left[-(t-y)^{2}\right] \\
& \times H_{m}\left[\left(\frac{2 \omega_{1}}{\omega_{1}+\omega_{2}}\right)^{1 / 2} t\right] \\
& \times H_{n}\left[\left(\frac{2 \omega_{2}}{\omega_{1}+\omega_{2}}\right)^{1 / 2} t-\left(\frac{\omega_{2}}{\omega_{0}}\right)^{1 / 2}\right] d t,
\end{aligned}
$$

where

$$
\begin{aligned}
N_{m n}= & \left(\omega_{1} \omega_{2}\right)^{1 / 4}\left[\left(\omega_{1}+\omega_{2}\right) 2^{m+n-1} m ! n !\right]^{-1 / 2} \\
& \times \exp \left(-y^{2} \omega_{1} / \omega_{2}\right)
\end{aligned}
$$

and 


$$
y=\omega_{2} /\left[2 \omega_{0}\left(\omega_{1}+\omega_{2}\right)\right]^{1 / 2} \text {. }
$$

The integral in Eq. (4) is of the same form as the integral defined in the Appendix. A direct use of Eq. (A8) yields a closed-form expression for the Franck-Condon integral:

$$
\begin{aligned}
\langle m \mid n\rangle\rangle= & N_{m n} \sum_{k=0}^{[m, n]}\left(\begin{array}{c}
m \\
k
\end{array}\right)\left(\begin{array}{l}
n \\
k
\end{array}\right)\left(\frac{\omega_{1}-\omega_{2}}{\omega_{1}+\omega_{2}}\right)^{(n-k) / 2} \\
& \times\left(\frac{\omega_{2}-\omega_{1}}{\omega_{1}+\omega_{2}}\right)^{(m-k) / 2}\left[\frac{4\left(\omega_{1} \omega_{2}\right)^{1 / 2}}{\omega_{1}+\omega_{2}}\right]^{k} ! \\
& \times H_{m-k}\left[\left(\frac{\omega_{1} \omega_{2}^{2}}{\omega_{0}\left(\omega_{2}^{2}-\omega_{1}^{2}\right)}\right)^{1 / 2}\right] \\
& \times H_{n-k}\left[-\left(\frac{\omega_{1}^{2} \omega_{2}}{\omega_{0}\left(\omega_{1}^{2}-\omega_{2}^{2}\right)}\right)^{1 / 2}\right] .
\end{aligned}
$$

This expression has been obtained, using various procedures, by a number of investigators. ${ }^{6-8}$ It is interesting to note that for the two special cases, $m=0$ or $n=0$, the above sum reduces to a single term containing only one Hermite polynomial. [We note parenthetically that the above sum (6) also reduces to a single term for the case of equal frequency oscillators.] From the recursion relation for Hermite polynomials (A3), it follows:

$$
\begin{aligned}
\langle 0 \mid n+2\rangle\rangle= & \left.\frac{-\omega_{1}}{\omega_{1}+\omega_{2}}\left[\frac{2 \omega_{2}}{\omega_{0}(n+2)}\right]^{1 / 2}\langle 0 \mid n+1\rangle\right\rangle \\
& \left.-\left(\frac{\omega_{1}-\omega_{2}}{\omega_{1}+\omega_{2}}\right)\left(\frac{n+1}{n+2}\right)^{1 / 2}\langle 0 \mid n\rangle\right\rangle, \\
\langle m+2 \mid 0\rangle\rangle= & \left.\frac{\omega_{2}}{\omega_{1}+\omega_{2}}\left[\frac{2 \omega_{1}}{\omega_{0}(m+2)}\right]^{1 / 2}\langle m+1 \mid 0\rangle\right\rangle \\
& \left.+\left(\frac{\omega_{1}-\omega_{2}}{\omega_{1}+\omega_{2}}\right)\left(\frac{m+1}{m+2}\right)^{1 / 2}\langle m \mid 0\rangle\right\rangle .
\end{aligned}
$$

In fact, on using the one-term expressions for $\langle 0 \mid n\rangle\rangle$ and $\langle m \mid 0\rangle\rangle$, Eq. (6) can be rewritten as

$$
\begin{aligned}
\langle m \mid n\rangle\rangle= & \sum_{k=0}^{[m, n]}\left[\frac{2\left(\omega_{1} \omega_{2}\right)^{1 / 2}}{\omega_{1}+\omega_{2}}\right]^{k}\left(\begin{array}{c}
m \\
k
\end{array}\right)^{1 / 2}\left(\begin{array}{l}
n \\
k
\end{array}\right)^{1 / 2} \\
& \times \frac{\langle m-k \mid 0\rangle\rangle\langle 0 \mid n-k\rangle\rangle}{\langle 0 \mid 0\rangle\rangle} .
\end{aligned}
$$

Equivalent expressions have been obtained previously by Manneback ${ }^{6}$ and Smith. ${ }^{9}$ It is remarkable-and this fact has apparently not been appreciated earlier in the literaturethat the complete Franck-Condon matrix $\langle m \mid n\rangle\rangle$ can be determined using Eqs. (7), (8), and (9) from the mere knowledge of either $\langle 0 \mid 0\rangle\rangle$ and $\langle 0 \mid 1\rangle)$, or $\langle 0 \mid 0\rangle\rangle$ and $\langle 1 \mid 0\rangle\rangle$.

\section{MATRIX ELEMENTS OF SOME FUNCTIONS}

Now let us consider the two-center matrix elements of $x^{l}, \exp (-2 c x)$, and $\exp \left(-c x^{2}\right)$ in the harmonic oscillator basis. Various matrix elements can be written in terms of integrals of the form

$$
\begin{aligned}
& I[f(t) ; m, n ; a, b, y, z] \\
& \quad=\int_{-\infty}^{\infty} f(t) \exp \left[-(t-y)^{2}\right] H_{m}(a t) H_{n}(b t-z) d t,
\end{aligned}
$$

which is derived in detail for the case $f(t)=1$ in the Appen$\operatorname{dix}$ [see, for example, Eq. (A8)].

\section{A. Powers of the coordinate $x$}

The required matrix elements can be written as

$$
\begin{aligned}
\left.\left\langle m\left|x^{l}\right| n\right\rangle\right\rangle= & \frac{N_{m n}}{\pi^{1 / 2}}\left(\frac{2 \omega_{0} r^{2}}{\omega_{1}+\omega_{2}}\right)^{l / 2} \\
& \times \int_{-\infty}^{\infty} t^{l} \exp \left[-(t-y)^{2}\right] \\
& \times H_{m}(a t) H_{n}(b t-z) d t,
\end{aligned}
$$

where

$$
\begin{aligned}
& a=\left[2 \omega_{1} /\left(\omega_{1}+\omega_{2}\right)\right]^{1 / 2}, \quad b=\left[2 \omega_{2} /\left(\omega_{1}+\omega_{2}\right)\right]^{1 / 2}, \\
& \text { and } z=\left(\omega_{2} / \omega_{0}\right)^{1 / 2} .
\end{aligned}
$$

$N_{m n}$ and $y$ are defined in Eq. (5). Using Eq. (A13), the matrix elements of $x^{l}$ can be written as a sum of FranckCondon integrals,

$$
\begin{aligned}
\left.\left\langle m\left|x^{l}\right| n\right\rangle\right\rangle= & {\left[\frac{\omega_{0} r^{2}}{2\left(\omega_{1}+\omega_{2}\right)}\right]^{l / 2} l ! } \\
& \times \sum_{p=0}^{[m, l]} \sum_{q=0}^{[n, l-p]}\left[\frac{m ! n !}{(m-p) !(n-q) !}\right]^{1 / 2} \\
& \times \frac{\left(4 \omega_{1}\right)^{p / 2}\left(4 \omega_{2}\right)^{q / 2}}{\left(\omega_{1}+\omega_{2}\right)^{(p+q) / 2} p ! q !} \\
& \times \frac{(-i)^{l-p-q}}{(l-p-q) !} H_{l-p-q}(i y) \\
& \times\langle m-p \mid n-q\rangle\rangle .
\end{aligned}
$$

This expression was obtained earlier by Morales et al., ${ }^{5}$ though there appears to be an error in the constants of their expression.

\section{B. Exponential function $\exp (-2 c x)$}

Here, in obtaining the relevant integral, we follow exactly the same steps as in the Appendix, Eqs. (A4)-(A6), except there now is an extra factor of $\exp (-2 c t)$ in the integrand. It leads to the following result for the integral:

$$
\begin{aligned}
I[\exp ( & -2 c t) ; m, n ; a, b, y, z] \\
= & \pi^{1 / 2} \exp \left(c^{2}-2 c y\right)\left(\frac{\partial}{\partial t_{2}}\right)_{t_{2}=0}^{n}\left(\frac{\partial}{\partial t_{1}}\right)_{t_{1}=0}^{m} \\
& \quad \times \exp \left\{-\left(1-a^{2}\right) t_{1}^{2}-\left(1-b^{2}\right) t_{2}^{2}\right. \\
& \left.+2 a(y-c) t_{1}+2[b(y-c)-z] t_{2}+2 a b t_{1} t_{2}\right\} .
\end{aligned}
$$

Except for the constant $\exp \left(c^{2}-2 c y\right)$, Eq. (14) looks just like Eq. (A6), and we can immediately write down the final result:

$$
\begin{aligned}
& I[\exp (-2 c t) ; m, n ; a, b, y, z] \\
& \quad=\exp \left(c^{2}-2 c y\right) I[1 ; m, n ; a, b, y-c, z] .
\end{aligned}
$$

The matrix element $\langle m|\exp (-2 c x)| n\rangle\rangle$ is related to $I[\exp (-2 c t) ; m, n ; a, b, y, z]$ and by using Eq. (15) one can write 


$$
\begin{aligned}
\langle m|\exp (-2 c x)| n\rangle\rangle= & N_{m n} \exp \left(\alpha^{2}-2 \alpha y\right) \\
& \times I[1 ; m, n ; a, b, y-\alpha, z] / \pi^{1 / 2},
\end{aligned}
$$

where $\alpha=c\left[\left(2 \omega_{0} r^{2}\right) /\left(\omega_{1}+\omega_{2}\right)\right]^{1 / 2}$ is introduced by a change of the integration variable from $x$ to the dimensionless $t=x\left(\omega_{1}+\omega_{2}\right)^{1 / 2} /\left(2 \omega_{0} r^{2}\right)^{1 / 2}$.

\section{Gaussian function $\exp \left(-c x^{2}\right)$}

The case $f(t)=\exp \left(-c t^{2}\right)$ can be worked out in a fashion very similar to the case of the exponential above. Following the same steps as Eqs. (A4) and (A5), we obtain $I\left[\exp \left(-c t^{2}\right) ; m, n ; a, b, y, z\right]$

$$
\begin{aligned}
= & \beta \exp \left(-c \beta^{2} y^{2}\right)\left(\frac{\partial}{\partial t_{2}}\right)_{t_{2}=0}^{n}\left(\frac{\partial}{\partial t_{1}}\right)_{t_{1}=0}^{m} \\
& \times \exp \left(-t_{1}^{2}-t_{2}^{2}-2 z t_{2}-\beta^{2} y^{2}\right) \\
& \times \int_{-\infty}^{\infty} \exp \left[-t^{2}-2\left(a \beta t_{1}+b \beta t_{2}+\beta y\right) t\right] d t,
\end{aligned}
$$

where $\beta=(1+c)^{-1 / 2}$.

The expression in Eq. (17) has essentially the same form as Eq. (A5), and we can immediately write down the result by inspection,

$I\left[\exp \left(-c t^{2}\right) ; m, n ; a, b, y, z\right]$

$$
=\beta \exp \left(-c \beta^{2} y^{2}\right) I[1 ; m, n ; a \beta, b \beta, y \beta, z] \text {. }
$$

The matrix elements $\left.\left\langle m\left|\exp \left(-c x^{2}\right)\right| n\right\rangle\right\rangle$ are related to the above integral by

$$
\begin{aligned}
\left.\left\langle m\left|\exp \left(-c x^{2}\right)\right| n\right\rangle\right\rangle= & N_{m n} \gamma \exp \left[-\left(1-\gamma^{2}\right) y^{2}\right] \\
& \times I[1 ; m, n ; \gamma a, \gamma b, \gamma y, z] / \pi^{1 / 2},
\end{aligned}
$$

where $\gamma=\left[1+2 c \omega_{0} r^{2} /\left(\omega_{1}+\omega_{2}\right)\right]^{-1 / 2}$ is once again introduced by the change of integration variable.

Summarizing this section, the integral for $f(t)=t^{l}$ can be written as a finite sum of integrals for $f(t)=1$. The integrals for the exponential and Gaussian functions can each be obtained by a simple scaling of an $f(t)=1$ integral.

\section{RECURSION RELATIONS}

Four-term recursion relations among Franck-Condon overlap integrals were derived by Ansbacher ${ }^{7}$ and, in equivalent form, by Manneback. ${ }^{6}$ These recursion relations are special cases of the more general recursion relations, to be obtained below, for the integral $I\left[t^{l} ; m, n ; a, b, y, z\right]$. In the preceding section it was shown that the integrals for the exponential and Gaussian functions could be written in terms of $I[1 ; m, n ; a, b, y, z]$ so that the recursion relations for the integrals for these functions are also obtained from the recursion relations for $I\left[t^{l} ; m, n ; a, b, y, z\right]$.

For brevity, define $I_{l}(m, n) \equiv I\left[t^{l} ; m, n ; a, b, y, z\right]$ in the following discussion. Thus,

$I_{l}(m, n)=\int_{-\infty}^{\infty} \exp \left[-(t-y)^{2}\right] t^{l} H_{m}(a t) H_{n}(b t-z) d t$

$$
=2 a I_{l+1}(m-1, n)-2(m-1) I_{l}(m-2, n)
$$

or equivalently,

$$
2 a I_{l+1}(m, n)=I_{l}(m+1, n)+2 m I_{l}(m-1, n) .
$$

In going from Eq. (20) to Eq. (21), the recursion relation for Hermite polynomials (A3) was applied to $H_{m}(a t)$. Using the recursion relation for Hermite polynomials on the $H_{n}(b t-z)$ term in Eq. (20), on the other hand, gives

$$
\begin{aligned}
2 b I_{l+1}(m, n)= & I_{l}(m, n+1)+2 z I_{l}(m, n) \\
& +2 n I_{l}(m, n-1) .
\end{aligned}
$$

It is important to note that from Eq. (22) or (23), the complete matrix of any power of $t$ (for example, $t^{l}$ ) can be obtained from the knowledge of the matrix of the next lower power (namely, $t^{l-1}$ ). We reemphasize that the matrix of Franck-Condon overlap integrals can be completely determined from a mere knowledge of only two matrix elements, $\langle 0 \mid 0\rangle\rangle$ and $\langle 0 \mid 1\rangle\rangle$ (or $\langle 0 \mid 0\rangle\rangle$ and $\langle 1 \mid 0\rangle\rangle)$, so, in principle, the complete matrix of any power of $x$ can be built up from only two overlap matrix elements.

Using, in the integrands of the terms on the left-hand sides of Eqs. (22) and (23),

$$
\begin{aligned}
t \exp \left[-(t-y)^{2}\right]= & -\frac{1}{2}\left(\frac{d}{d t}\right) \exp \left[-(t-y)^{2}\right] \\
& +y \exp \left[-(\mathrm{t}-\mathrm{y})^{2}\right]
\end{aligned}
$$

then performing integration by parts and the necessary derivatives we obtain

$$
\begin{aligned}
a l I_{l-1}(m, n)= & I_{l}(m+1, n)-2 n a b I_{l}(m, n-1) \\
& +2 m\left(1-a^{2}\right) I_{l}(m-1, n)-2 a y I_{l}(m, n)
\end{aligned}
$$

and

$$
\begin{aligned}
b l I_{l-1}(m, n)= & I_{l}(m, n+1)-2 m a b I_{l}(m-1, n) \\
& +2 n\left(1-b^{2}\right) I_{l}(m, n-1) \\
& -2(b y-z) I_{l}(m, n) .
\end{aligned}
$$

The recursion relations for these integrals can easily be adapted to the matrix elements $\left.\left\langle\boldsymbol{m}\left|\boldsymbol{x}^{I}\right| n\right\rangle\right\rangle$, using Eq. (11):

$$
\begin{aligned}
&\left.2 \operatorname{lr}\left(\omega_{1} \omega_{0}\right)^{1 / 2}\left\langle\mathrm{~m}\left|\mathrm{x}^{l-1}\right| \mathrm{n}\right\rangle\right\rangle \\
&= {\left.[2(m+1)]^{1 / 2}\left(\omega_{1}+\omega_{2}\right)\left\langle m+1\left|x^{l}\right| n\right\rangle\right\rangle } \\
&\left.-\left(8 n \omega_{1} \omega_{2}\right)^{1 / 2}\left\langle m\left|x^{l}\right| n-1\right\rangle\right\rangle \\
&\left.+(2 m)^{1 / 2}\left(\omega_{2}-\omega_{1}\right)\left\langle m-1\left|x^{l}\right| n\right\rangle\right\rangle \\
&\left.-\left(\omega_{1} / \omega_{0}\right)^{1 / 2} 2 \omega_{2}\left\langle m\left|x^{l}\right| n\right\rangle\right\rangle, \\
&\left.2 \operatorname{lr}\left(\omega_{2} \omega_{0}\right)^{1 / 2}\left\langle m\left|x^{l-1}\right| n\right\rangle\right\rangle \\
&\left.=[2(n+1)]^{1 / 2}\left(\omega_{1}+\omega_{2}\right)\left\langle m\left|x^{l}\right| n+1\right\rangle\right\rangle \\
&\left.\quad-\left(8 m \omega_{1} \omega_{2}\right)^{1 / 2}\left\langle m-1\left|x^{l}\right| n\right\rangle\right\rangle \\
&\left.+(2 n)^{1 / 2}\left(\omega_{1}-\omega_{2}\right)\left\langle m\left|x^{l}\right| n-1\right\rangle\right\rangle \\
&\left.+\left(\omega_{2} / \omega_{0}\right)^{1 / 2} 2 \omega_{1}\left\langle m\left|x^{l}\right| n\right\rangle\right\rangle .
\end{aligned}
$$

Equations (26) and (27) are generalized forms of Ansbacher's ${ }^{7}$ recursion relations for Franck-Condon overlap integrals which are obtained by letting $l=0$. 
Equations (22) and (23) can be adapted to the integrals for the exponential and Gaussian functions by multiplying the equations by $(-2 c)^{l} / l !$ or $(-c t)^{l} / l !$, respectively, and then summing over $l$. This yields, for the exponential case (suppressing the constants $a, b, y$, and $z$ ):

$$
\begin{aligned}
& 2 a I[t \exp (-2 c t) ; m, n] \\
& =I[\exp (-2 c t) ; m+1, n] \\
& \quad+2 m I[\exp (-2 c t) ; m-1, n], \\
& 2 b I[t \exp (-2 c t) ; m, n] \\
& =I[\exp (-2 c t) ; m, n+1] \\
& \quad+2 z I[\exp (-2 c t) ; m, n] \\
& \quad+2 n I[\exp (-2 c t) ; m, n-1] .
\end{aligned}
$$

Note that these equations relate the $f(t)=\exp (-2 c t)$ integral to the $f(t)=t \exp (-2 c t)$ integral. Analogous relations for the Gaussian case can be obtained in a similar manner.

Equations (24) and (25) [or Eqs. (26) and (27)] can be similarly adapted for integrals for the exponential and Gaussian functions. For the exponential case, Eqs. (24) and (25) are transformed into

$$
\begin{aligned}
& 2 a(y-c) I[\exp (-2 c t) ; m, n] \\
&=I {[\exp (-2 c t) ; m+1, n] } \\
& \quad-2 n a b I[\exp (-2 c t) ; m, n-1] \\
& \quad+2 m\left(1-a^{2}\right) I[\exp (-2 c t) ; m-1, n], \\
& 2[b(y-c)-z] I[\exp (-2 c t) ; m, n] \\
&=I {[\exp (-2 c t) ; m, n+1] } \\
&-2 m a b I[\exp (-2 c t) ; m-1, n] \\
&+2 n\left(1-b^{2}\right) I[\exp (-2 c t) ; m, n-1] .
\end{aligned}
$$

Equations (30) and (31) could have been obtained by an alternative method using the results of Sec. III B, in which it was shown that the integrals for the exponential and Gaussian functions were related by simple scaling to the $f(t)=1$ integral. Thus setting $l=0$ in Eqs. (24) and (25) or in Eqs. (26) and (27), and using Eq. (15) for the scaling property of the exponential case, the recursion relations (30) and (31) are immediately obtained. This alternative procedure provides a self-consistent check on the present results. A similar check can be easily verified for the Gaussian case using the scaling property (18).

Equations (22) and (23) or Eqs. (24) and (25) can be combined to eliminate the integral on the left side in each case, and obtain a five-term recursion relation valid for the matrix elements of powers of $x$. It turns out that the recursion relation thus obtained is very general; since it is good for any power of $x$, it will be valid for any analytic function of $x$ which can be expanded in a power series. Thus,

$$
\begin{aligned}
\langle m|f(x)| n\rangle\rangle= & \left(\frac{\omega_{0}}{2 \omega_{1}}\right)^{1 / 2}\left\{(m+1)^{1 / 2}\langle m+1|f(x)| n\rangle\right\rangle \\
& \left.\left.+m^{1 / 2}\langle m-1|f(x)| n\rangle\right)\right\}-\left(\frac{\omega_{0}}{2 \omega_{2}}\right)^{1 / 2} \\
& \times\left\{(n+1)^{1 / 2}\langle m|f(x)| n+1\rangle\right\rangle \\
& \left.\left.+n^{1 / 2}\langle m|f(x)| n-1\rangle\right\rangle\right\}
\end{aligned}
$$

where $f(x)$ can be a power, exponential, Gaussian, trigonometric function, etc.

\section{DISCUSSION AND CONCLUSIONS}

For the special case of equal frequency oscillators $\left(\omega_{1}=\omega_{2}\right)$, expression (6) for the Franck-Condon overlap integral reduces to a series which can be identified as a representation of an associated Laguerre polynomial. ${ }^{10}$ Explicitly, for $\omega_{1}=\omega_{2}=\omega$,

$$
\begin{aligned}
\langle m \mid n\rangle\rangle= & (-1)^{n-m}\left[\frac{m !}{n !}\left(\frac{\omega}{2 \omega_{0}}\right)^{n-m}\right. \\
& \left.\times \exp \left(-\frac{\omega}{2 \omega_{0}}\right)\right]^{1 / 2} L_{m}^{n-m}\left(\frac{\omega}{2 \omega_{0}}\right) .
\end{aligned}
$$

During their numerical evaluation of integrals of the form $\left.\left\langle m\left|x^{l}\right| n\right\rangle\right\rangle$ for the first positive system of $\mathrm{N}_{2}$, Fraser ${ }^{11}$ and Nicholls and Jarmain ${ }^{12}$ observed that under certain conditions the following equality nearly holds:

$$
\frac{\left.\left\langle m\left|x^{2}\right| n\right\rangle\right\rangle}{\langle m|x| n\rangle\rangle} \approx \frac{\langle m|x| n\rangle\rangle}{\left.\left\langle m\left|x^{0}\right| n\right\rangle\right\rangle} \text {. }
$$

That this should be true is easily seen by using the recursion relation (22). For the case $\omega \gg \omega_{0}$ and $\left(\omega / \omega_{0}\right) \gg m, n$ [which are equivalent ${ }^{11,12}$ to the conditions necessary for equality (34) to hold], it is readily seen that the ratios in Eq. (34) are approximately equal to $\frac{1}{2} r$, independent of $m$ or $n$.

In order to illustrate the results of the recursion relations derived above, we have numerically evaluated the Franck-Condon factors for the $A^{1} \Sigma_{u}^{+}-X^{1} \Sigma_{g}^{+}$and the $B^{1} \Pi_{u}-X^{1} \Sigma_{g}^{+}$systems of ${ }^{7} \mathrm{Li}_{2}$ using Eqs. (7), (8), and (9). The harmonic oscillators representing the potential curves of the $X, A$, and $B$ electronic states have frequencies 351.43 , 255.45 , and $269.69 \mathrm{~cm}^{-1}$, respectively, and potential minimum at $2.672,3.107$, and $2.936 \AA$, respectively. It is easy to verify that the first five vibrational levels of the above three simple harmonic oscillators have the same energy levels, within $5 \%$, as the actual vibrational energy levels of the three electronic states, indicating that the harmonic oscillator approximation is reasonable for these levels. The computed numbers for Franck-Condon factors are compared, in Table I, with the experimentally obtained values of these factors for the above transitions in $\mathrm{Li}_{2}$ by Hessel and coworkers. ${ }^{14,15}$ To make comparison easy, we use a double ket notation, $|m\rangle\rangle$, to indicate the $m$ th vibrational level of the ground state (analogous to the double prime, $v^{\prime \prime}$, notation of Hessel) and a single ket notation, $|n\rangle$, to indicate the $n$th vibrational level of the excited $A$ or $B$ states (analogous to the single prime, $v^{\prime}$, notation of Hessel). The results shown in Table $I$ indicate that the agreement between computed factors, $\left.|\langle m \mid n\rangle\rangle\right|^{2}$, and experimental values is not encouraging even for the low vibrational levels where the harmonic oscillator is supposed to be a good approximation. However, we note that the agreement becomes reasonable when the designation of the vibrational quantum numbers of two relevant levels are interchanged, that is, when the experimental $\left.|\langle m \mid n\rangle\rangle\right|^{2}$ is compared with $\mid\left\langle\left.\langle m \mid n\rangle\right|^{2}\right.$. For ease of comparison, we have displayed $\left.|\langle m \mid n\rangle\rangle\right|^{2}$ and $\mid\left\langle\left.\langle m \mid n\rangle\right|^{2}\right.$ side-byside in Table $\mathrm{I}$. We do not yet understand the reason for this puzzling observation. 
TABLE I. A comparison of the Franck-Condon factors for the $A^{1} \Sigma_{u}^{+}-X^{1} \Sigma_{\mathrm{g}}^{+}$system (multiplied by $10^{3}$ ) and the $B^{1} \Pi_{u}-X^{1} \Sigma_{g}^{+}$system (multiplied by $10^{4}$ ) of ${ }^{7} \mathrm{Li}_{2}$. A double (|) $\rangle$ ) and a single $(|\rangle)$ ket notation refers to the vibrational levels of the ground and the excited electronic state, respectively.

\begin{tabular}{|c|c|c|c|c|c|c|}
\hline \multirow{3}{*}{$\begin{array}{c}\text { Vibrational } \\
\text { quantum } \\
\text { number } \\
m, n\end{array}$} & \multicolumn{3}{|c|}{$A^{1} \Sigma_{u}^{+}-X^{1} \Sigma_{g}^{+}$} & \multicolumn{3}{|c|}{$B^{1} \Pi_{u}-X^{1} \Sigma_{g}^{+}$} \\
\hline & \multicolumn{3}{|c|}{ experiment (Ref. 14) } & \multicolumn{3}{|c|}{ experiment ( Ref. 15) } \\
\hline & $\left.|\langle m \mid n\rangle\rangle\right|^{2}$ & $\left.|\langle m \mid n\rangle\rangle\right|^{2}$ & $\mid\left\langle\left.\langle m \mid n\rangle\right|^{2}\right.$ & $\left.|\langle m \mid n\rangle\rangle\right|^{2}$ & $\left.|\langle m \mid n\rangle\rangle\right|^{2}$ & $\mid\left\langle\left.\langle m \mid n\rangle\right|^{2}\right.$ \\
\hline 0,0 & 53 & 52 & 53 & 3267 & 3188 & 3267 \\
\hline 0,1 & 131 & 176 & 180 & 3149 & 3827 & 4104 \\
\hline 0,2 & 182 & 270 & 278 & 1961 & 2103 & 2065 \\
\hline 0,3 & 187 & 250 & 254 & 969 & 698 & 507 \\
\hline 0,4 & 158 & 156 & 153 & 413 & 156 & 55 \\
\hline 1,0 & 180 & 134 & 131 & 4104 & 3340 & 3149 \\
\hline 1,1 & 191 & 197 & 191 & 39 & 77 & 39 \\
\hline 1,2 & 78 & 58 & 54 & 844 & 1511 & 2042 \\
\hline 1,3 & 4 & 9 & 12 & 1692 & 2711 & 3127 \\
\hline 1,4 & 15 & 134 & 145 & 1516 & 1657 & 1395 \\
\hline 2,0 & 277 & 187 & 182 & 2065 & 2008 & 1961 \\
\hline 2,1 & 54 & 79 & 78 & 2042 & 942 & 844 \\
\hline 2,2 & 13 & 15 & 13 & 1110 & 1345 & 1110 \\
\hline 2,3 & 9 & 127 & 120 & 0.5 & 63 & 329 \\
\hline 2,4 & 88 & 56 & 51 & 622 & 1834 & 2826 \\
\hline 3,0 & 254 & 190 & 188 & 507 & 918 & 969 \\
\hline 3,1 & 12 & 3 & 4 & 3127 & 1884 & 1692 \\
\hline 3,2 & 120 & 98 & 90 & 329 & 1 & 0.5 \\
\hline 3,3 & 46 & 45 & 46 & 1391 & 1508 & 1391 \\
\hline 3,4 & 2 & 25 & 20 & 423 & 303 & 28 \\
\hline 4,0 & 153 & 157 & 158 & 55 & 358 & 413 \\
\hline 4,1 & 145 & 18 & 15 & 1395 & 1585 & 1516 \\
\hline 4,2 & 51 & 90 & 89 & 2826 & 711 & 622 \\
\hline 4,3 & 20 & 4 & 2 & 28 & 550 & 423 \\
\hline 4,4 & 84 & 92 & 84 & 800 & 661 & 800 \\
\hline
\end{tabular}

To summarize, we have obtained explicit expressions for the matrix elements of $x^{l}, \exp (-2 c x)$, and $\exp \left(-c x^{2}\right)$, $x$ being the internuclear separation, in the two-center simple harmonic basis. It is shown that in principle, the complete matrices of combinations of these functions could be determined in terms of only the lowest two Franck-Condon overlap matrix elements. Furthermore, a very general five-term recursion relation (32) is obtained which is valid for the matrix elements of any analytic function of $x$.

\section{ACKNOWLEDGMENTS}

It is a pleasure to thank Professor H. B. Schlegel for bringing some useful references to our attention. This research is supported by the Air Force Office of Scientific Research under Grant No. AFOSR-84-0143.

\section{APPENDIX}

The integral

$$
\begin{aligned}
& I[f(t) ; m, n ; a, b, y, z] \\
& \quad=\int_{-\infty}^{\infty} f(t) \exp \left[-(t-y)^{2}\right] H_{m}(a t) H_{n}(b t-z) d t
\end{aligned}
$$

with $a, b, y$, and $z$ constant, is very useful in the evaluation of the Franck-Condon factors, and the matrix elements of powers of the coordinate $x$. The matrix elements of exponential and Gaussian functions, $\exp (-2 c x)$ and $\exp \left(-c x^{2}\right)$, respectively, can also be easily obtained with only slight modifications in the solution of the integral for the case $f(t)=1$. To this end, a detailed derivation of the integral, $I(1 ; m, n ; a, b, y, z) \equiv I_{0}$, is given in this Appendix. Some formulas, useful in the evaluation of the integral, and in obtaining recursion relations for it are presented first.

From the generating function of Hermite polynomials,

$$
\sum_{n=0}^{\infty} H_{n}(x) t^{n} / n !=\exp \left(-t^{2}+2 x t\right),
$$

the following representation for Hermite polynomials is obtained:

$$
H_{n}(B / A)=A^{-n}\left(\frac{\partial}{\partial t}\right)_{t=0}^{n} \exp \left(-A^{2} t^{2}+2 B t\right) .
$$
mials is

The three-term recursion relation for Hermite polyno-

$$
2 x H_{n}(x)=H_{n+1}(x)+2 n H_{n-1}(x) .
$$

The integral to be evaluated is

$$
I_{0}=\int_{-\infty}^{\infty} \exp \left[-(t-y)^{2}\right] H_{m}(a t) H_{n}(b t-z) d t .
$$

Using Eq. (A2) for the Hermite polynomials in Eq. (A4) we obtain

$$
\begin{aligned}
I_{0}= & \left(\frac{\partial}{\partial t_{1}}\right)_{t_{1}=0}^{m}\left(\frac{\partial}{\partial t_{2}}\right)_{t_{2}=0}^{n} \exp \left(-t_{1}^{2}-t_{2}^{2}-2 z t_{2}-y^{2}\right) \\
& \times \int_{-\infty}^{\infty} \exp \left[-t^{2}+2\left(a t_{1}+b t_{2}+y\right) t\right] d t \quad \text { (A5) }
\end{aligned}
$$




$$
\begin{aligned}
= & \pi^{1 / 2}\left(\frac{\partial}{\partial t_{1}}\right)_{t_{1}=0}^{m}\left(\frac{\partial}{\partial t_{2}}\right)_{t_{2}=0}^{n} \exp \left[-\left(1-a^{2}\right) t_{1}^{2}\right. \\
& \left.-\left(1-b^{2}\right) t_{2}^{2}+2 a b t_{1} t_{2}+2 a y t_{1}+2(b y-z) t_{2}\right] .
\end{aligned}
$$

(A6)

Carrying out the $t_{2}$ derivatives, and using the definition (A2) of the Hermite polynomials leads to

$$
\begin{aligned}
I_{0}= & \pi^{1 / 2} \sum_{k=0}^{n}\left(\begin{array}{l}
n \\
k
\end{array}\right)\left(1-b^{2}\right)^{(n-k) / 2} H_{n-k}\left[\frac{b y-z}{\left(1-b^{2}\right)^{1 / 2}}\right] \\
& \times \sum_{j=0}^{m}\left(\begin{array}{c}
m \\
j
\end{array}\right)\left(\frac{\partial}{\partial t_{1}}\right)_{t_{1}=0}^{j}\left(2 a b t_{1}\right)^{k}\left(\frac{\partial}{\partial t_{1}}\right)_{t_{1}=0}^{m-j} \\
& \times \exp \left[-\left(1-a^{2}\right) t_{1}^{2}+2 a y t_{1}\right] \\
= & \pi^{1 / 2} \sum_{k=0}^{[m, n]}\left(\begin{array}{l}
n \\
k
\end{array}\right)\left(\begin{array}{c}
m \\
k
\end{array}\right)\left(1-b^{2}\right)^{(n-k) / 2}\left(1-a^{2}\right)^{(m-k) / 2} \\
& \times(2 a b)^{k} k ! H_{m-k}\left[\frac{a y}{\left(1-a^{2}\right)^{1 / 2}}\right] \\
& \times H_{n-k}\left[\frac{b y-z}{\left(1-b^{2}\right)^{1 / 2}}\right]
\end{aligned}
$$

since, on setting $t_{1}=0$, the only nonzero term in the $j$ sum is $j=k$. Thus the $k$ sum runs from zero to the smaller of $m$ or $n$, indicated by $[m, n]$.

We can evaluate $I\left[t^{l} ; m, n ; a, b, y, z\right] \equiv I_{l}$ by following the same procedure as above, through Eq. (A6), and using the standard integral, ${ }^{13}$

$$
\int_{-\infty}^{\infty} t^{l} \exp \left(-t^{2}+2 u t\right) d t=\pi^{1 / 2} \exp \left(u^{2}\right)(2 i)^{-l} H_{l}(i u) \text {. }
$$

We obtain

$$
\begin{aligned}
I_{l}= & (2 i)^{-1} \pi^{1 / 2}\left(\frac{\partial}{\partial t_{2}}\right)_{t_{2}=0}^{n}\left(\frac{\partial}{\partial t_{1}}\right)_{t_{1}=0}^{m} \\
& \times H_{l}(i u) A\left(t_{1}, t_{2}\right) \\
= & (2 i)^{-1} \pi^{1 / 2} \sum_{p=0}^{m} \sum_{q=0}^{n}\left(\begin{array}{c}
m \\
p
\end{array}\right)\left(\begin{array}{c}
n \\
q
\end{array}\right) \\
& \times\left[\left(\frac{\partial}{\partial t_{2}}\right)_{t_{2}=0}^{q}\left(\frac{\partial}{\partial t_{1}}\right)_{t_{1}=0}^{p} H(i u)\right] \\
& \times\left[\left(\frac{\partial}{\partial t_{2}}\right)_{t_{2}=0}^{n-q}\left(\frac{\partial}{\partial t_{1}}\right)_{t_{1}=0}^{m-p} A\left(t_{1}, t_{2}\right)\right],
\end{aligned}
$$

where

$$
\begin{gathered}
A\left(t_{1}, t_{2}\right)=\exp \left[-\left(1-a^{2}\right) t_{1}^{2}-\left(1-b^{2}\right) t_{2}^{2}\right. \\
\left.+2 a y t_{1}+2(b y-z) t_{2}+2 a b t_{1} t_{2}\right], \\
u=a t_{1}+b t_{2}+y \text { and } i=(-1)^{1 / 2} .
\end{gathered}
$$

Using Eq. (A6) and

$$
\begin{aligned}
& \left(\frac{\partial}{\partial t_{2}}\right)_{t_{2}=0}^{q}\left(\frac{\partial}{\partial t_{1}}\right)_{t_{1}=0}^{p} H_{l}(i u) \\
& \quad=\frac{l ! a^{p} b^{q}(2 i)^{p+q}}{(l-p-q) !} H_{l-p-q}(i y) ; p+q \leqslant l
\end{aligned}
$$

we obtain the final result:

$$
\begin{aligned}
& I\left[t^{l} ; m, n ; a, b, y, z\right] \\
&= \sum_{p=0}^{(m, l][n, l-p)} \sum_{q=0}^{\left(\begin{array}{c}
m \\
p
\end{array}\right)\left(\begin{array}{l}
n \\
q
\end{array}\right)} \\
& \times \frac{l ! a^{p} b^{q}}{(2 i)^{l-p-q}(l-p-q) !} H_{l-p-q}(i y) \\
& \times I[1 ; m-p, n-q ; a, b, y, z] .
\end{aligned}
$$

${ }^{1}$ G. Herzberg, Molecular Spectra and Molecular Structure, 2nd ed. (Van Nostrand, Princeton, 1950), Vol. I; J. I. Steinfeld, Molecules and Radiation, 2nd ed. (MIT, Cambridge, 1985).

${ }^{2}$ S. Waldenstrøm and K. Razi Naqvi, Chem. Phys. Lett. 85, 581 (1982).

${ }^{3}$ N. E. Kuz'menko, L. A. Kuznetsova, and Yu. Ya. Kuzyankov, Sov. Phys. Usp. 26, 425 (1983).

${ }^{4}$ K. Nishikawa, Int. J. Quantum Chem. 12, 859 (1977).

${ }^{5} \mathrm{~J}$. Morales, A. Palma, and M. Berrondo, Int. J. Quantum Chem. Symp. 18, 57 (1984).

${ }^{6} \mathrm{C}$. Manneback, Physica 17, 1001 (1951).

'F. Ansbacher, Z. Naturforsch. Teil A 14, 889 (1959).

${ }^{8}$ T. Terasaka and T. Matsushita, Chem. Phys. Lett. 80, 306 (1981).

${ }^{9}$ W. L. Smith, J. Phys. B 2, 1 (1969).

${ }^{10}$ I. S. Gradshteyn and I. M. Ryzhik, Table of Integrals, Series and Products (Academic, New York, 1980), p. 1037.

"'P. A. Fraser, Can. J. Phys. 32, 515 (1954).

${ }^{12}$ R. W. Nicholls and W. R. Jarmain, Proc. Phys. Soc. A 69, 253 (1955).

${ }^{13}$ I. S. Gradshteyn and I. M. Ryzhik, Ref. 10, p. 338.

${ }^{14}$ P. Kusch and M. M. Hessel, J. Chem. Phys. 67, 586 (1977).

${ }^{15}$ M. M. Hessel and C. R. Vidal, J. Chem. Phys. 70, 4439 (1979). 